PROCEEDINGS OF THE

AMERICAN MATHEMATICAL SOCIETY

Volume 137, Number 1, January 2009, Pages 155-159

S 0002-9939(08)09532-4

Article electronically published on July 10, 2008

\title{
ON A WEYL INEQUALITY OF OPERATORS IN BANACH SPACES
}

\author{
BERND CARL
}

(Communicated by N. Tomczak-Jaegermann)

\begin{abstract}
Let $s=\left(s_{n}\right)$ be an injective and surjective $s$-number sequence in the sense of Pietsch. We show for a Riesz-operator $T: X \rightarrow X$ acting on a (complex) Banach space the following Weyl inequality between geometric means of eigenvalues and $s$-numbers: For any $0<\delta \leq 1$ and all $n \in \mathbb{N}$,
\end{abstract}

$$
\left(\prod_{i=1}^{n}\left|\lambda_{i}(T)\right|\right)^{\frac{1}{n}} \leq c_{0}\left(1+\frac{1}{\delta} \ln \left(\frac{1}{\delta}\right)\right)\left(\prod_{i=1}^{\left[\frac{n}{1+\delta}\right]} s_{i}(T)\right)^{\frac{1}{\left.\frac{n}{1+\delta}\right]}},
$$

where $c_{0} \geq 1$ is an absolute constant. The proof rests on an elementary mixing multiplicativity of an arbitrary $s$-number sequence and a striking result of G. Pisier. The inequality is a contribution to the problem of estimating eigenvalues by $s$-numbers first started in a strong sense by H. König (1986, 2001).

\section{1. $s$-NUMBERS}

We recall some basic definitions and notions from Banach space theory and from $s$-numbers of operators. If $X$ is a Banach space, we denote by $X^{\prime}$ its dual Banach space and by $B_{X}$ and $\stackrel{\circ}{B}_{X}$ the closed and open unit balls of $X$, respectively. In what follows, $X, Y, Z$, etc., always denote Banach spaces, $\mathcal{L}(X, Y)$ is the set of (bounded linear) operators from $X$ into $Y$ equipped with the operator norm and $\mathcal{L}$ stands for the class of all operators between arbitrary Banach spaces.

We use the notion of an $s$-number sequence given in $\mathrm{P} 80 \mathrm{~b}$ or $\mathrm{P} 87$. A rule $s=\left(s_{n}\right): \mathcal{L} \rightarrow[0, \infty]$ assigning to every operator $T \in \mathcal{L}$ a non-negative scalar sequence $\left(s_{n}(T)\right)_{n \in \mathbb{N}}$ is called an s-number sequence if the following conditions are satisfied:

(S1) Monotonicity:

$$
\|T\|=s_{1}(T) \geq s_{2}(T) \geq \ldots \geq 0 \text { for } T \in \mathcal{L}(X, Y) .
$$

(S2) Additivity:

$$
s_{n+m-1}(S+T) \leq s_{m}(S)+s_{n}(T) \text { for } S, T \in \mathcal{L}(X, Y) .
$$

Received by the editors November 30, 2007.

2000 Mathematics Subject Classification. Primary 47B06, 47A75.

Key words and phrases. Weyl inequalities, eigenvalue estimates, approximation numbers, $s$-numbers. 
(S3) Ideal-property:

$s_{n}(S T R) \leq\|S\| s_{n}(T)\|R\|$ for $R \in \mathcal{L}\left(X_{0}, X\right), T \in \mathcal{L}(X, Y)$ and $S \in$ $\mathcal{L}\left(Y_{1}, Y_{0}\right)$.

(S4) Rank-property:

If $\operatorname{rank}(T)<n$, then $s_{n}(T)=0$.

(S5) Norming property:

$s_{n}\left(I: l_{2}^{n} \rightarrow l_{2}^{n}\right)=1$ for $n \in \mathbb{N}$,

where $I$ denotes the identity operator on the $n$-dimensional Hilbert space $l_{2}^{n}$.

Furthermore, we additionally need the following notions:

(J) An $s$-number sequence $s=\left(s_{n}\right)$ is called injective if, given any metric injection $J \in \mathcal{L}(Y, \tilde{Y})$, i.e. $\|J y\|=\|y\|$ for $y \in Y, s_{n}(T)=s_{n}(J T)$ for all $T \in \mathcal{L}(X, Y)$ and all Banach spaces $X$.

(S) An $s$-number sequence $s=\left(s_{n}\right)$ is called surjective if, given any metric surjection $Q \in \mathcal{L}(\tilde{X}, X)$, i.e. $Q\left(\stackrel{\circ}{B}_{\tilde{X}}\right)=\stackrel{\circ}{B}_{X}, s_{n}(T)=s_{n}(T Q)$ for all $T \in$ $\mathcal{L}(X, Y)$ and all Banach spaces $Y$.

(JS) An $s$-number sequence is called injective and surjective if it satisfies $(\mathrm{J})$ and $(\mathrm{S})$.

We note that on the class of Hilbert spaces there is only one $s$-number sequence satisfying the properties (S1) - (S5) that coincides with the singular numbers [P80b]. Basic examples for our purposes are the approximation numbers given by

$$
a_{n}(T):=\inf \{\|T-L\|: L \in \mathcal{L}(X, Y), \operatorname{rank}(L)<n\},
$$

the Gelfand numbers given by

$$
c_{n}(T):=\inf \left\{\left\|T J_{M}\right\|: M \subset X, \operatorname{codim}(M)<n\right\},
$$

where $J_{M}: M \rightarrow X$ is the natural embedding from a subspace $M$ of $X$ into $X$, and the Kolmogorov number given by

$$
d_{n}(T):=\inf \left\{\left\|Q_{N} T\right\|: N \subset Y, \operatorname{dim} N<n\right\},
$$

where $Q_{n}: Y \rightarrow Y / N$ defines the canonical quotient map from $Y$ onto the quotient space $Y / N$.

Moreover, we need also the following characterization of Gelfand and Kolmogorov numbers,

$$
c_{n}(T)=a_{n}\left(J_{\infty} T\right) \text { and } d_{n}(T)=a_{n}\left(T Q_{1}\right),
$$

where $J_{\infty}: Y \rightarrow l_{\infty}\left(B_{Y^{\prime}}\right)$ is the metric injection defined by $J_{\infty} y:=(\langle y, a\rangle)_{a \in B_{Y^{\prime}}}$ and with values in the space $l_{\infty}\left(B_{Y^{\prime}}\right)$ of bounded sequences and where $Q_{1}$ : $l_{1}\left(B_{X}\right) \rightarrow X$ is the metric surjection from the space of summable sequences $l_{1}\left(B_{X}\right)$ onto $X$, defined by $Q_{1}\left(\left(\xi_{x}\right)\right):=\sum_{x \in B_{X}} \xi_{x} x$.

Now we show an elementary but very useful multiplicativity property of an arbitrary $s$-number sequence that we call mixing multiplicativity.

(MI) Mixing multiplicativity: Let $s=\left(s_{n}\right)$ be an arbitrary $s$-number sequence. Then for $S \in \mathcal{L}(X, Y)$ and $T \in \mathcal{L}(Y, Z)$,

(i) $s_{n+m-1}(T S) \leq s_{n}(T) a_{m}(S)$ and $s_{n+m-1}(T S) \leq a_{n}(T) s_{m}(S)$. Moreover, if $s=\left(s_{n}\right)$ is injective, then

(ii) $s_{n+m-1}(T S) \leq c_{n}(T) s_{m}(S)$ and if $s=\left(s_{n}\right)$ is surjective, then

(iii) $s_{n+m-1}(T S) \leq s_{n}(T) d_{m}(S)$. 
Proof. We show the first inequality of (i); the second one can be similarly proved. To this end let $L \in \mathcal{L}(X, Y)$ be an operator with $\operatorname{rank}(L)<m$. Then the additivity (S2), the rank property (S4), and the ideal property (S3) of an $s$-number sequence immediately yield

$$
\begin{aligned}
s_{n+m-1}(T S) & =s_{n+m-1}(T(S-L)+T L) \\
& \leq s_{n}(T(S-L))+s_{m}(T L)=s_{n}(T(S-L)) \leq s_{n}(T)\|S-L\|,
\end{aligned}
$$

implying by definition of the approximation numbers the desired inequality $s_{n+m-1}(T S) \leq s_{n}(T) a_{m}(S)$.

The inequalities (ii) and (iii) follow from (i) and the characterization of Gelfand and Kolmogorov numbers, respectively. For example, (ii) follows from $s_{n+m-1}(T S)$ $=s_{n+m-1}\left(J_{\infty} T S\right) \leq a_{n}\left(J_{\infty} T\right) s_{m}(S)=c_{n}(T) s_{m}(S)$.

\section{A Weyl inequality}

For a Riesz operator $T \in \mathcal{L}(X)$ acting on a complex Banach space (cf. [K86, P87, CS90]) we assign an eigenvalue sequence $\left(\lambda_{n}(T)\right)$ as follows: The eigenvalues are arranged in an order of non-increasing absolute values and each eigenvalue is counted according to its algebraic multiplicity,

$$
\left|\lambda_{1}(T)\right| \geq\left|\lambda_{2}(T)\right| \geq \cdots \geq 0 .
$$

If $T$ possesses less than $n$ eigenvalues $\lambda$ with $\lambda \neq 0$, we put $\lambda_{n}(T)=\lambda_{n+1}(T)=$ $\cdots=0$. In this section we establish for an arbitrary injective and surjective $s$ number sequence a Weyl inequality for Riesz operators. The following Weyl inequality complements Weyl inequalities for $s$-numbers given in [K86, K01, KRT80, [P80a, [P87, H05] and [CHi07. In the sequel, $[x]$ denotes the integer part of $x$ for $1 \leq x<\infty$ and if $0<x \leq 1$ we put $[x]:=1$.

Theorem. Let $s=\left(s_{n}\right)$ be an injective and surjective s-number sequence. Then for any $0<\delta \leq 1$ there exists a constant $c(\delta) \geq 1$ such that for all (complex) Banach spaces $X$, all Riesz operators $T \in \mathcal{L}(X)$ and all $n \in \mathbb{N}$ the inequality

$$
\left(\prod_{i=1}^{n}\left|\lambda_{i}(T)\right|\right)^{\frac{1}{n}} \leq c(\delta)\left(\prod_{i=1}^{\left[\frac{n}{1+\delta}\right]} s_{i}(T)\right)^{\frac{1}{\left[\frac{n}{1+\delta}\right]}}
$$

holds. For the constant $c(\delta)$ we obtain the estimate $c(\delta) \leq c_{0}\left(1+\frac{1}{\delta} \ln \frac{1}{\delta}\right)$, where $c_{0} \geq 1$ is an absolute constant.

Proof. In order to prove the inequality we need a striking result of Pisier [Pi89a], Pi89b concerning the existence of isomorphisms between arbitrary $n$-dimensional Banach spaces and $n$-dimensional Hilbert spaces and the mixing multiplicativity of an injective and surjective $s$-number sequence.

Pisier's isomorphism states the following:

For each $\alpha>\frac{1}{2}$, there is a constant $b(\alpha)$ such that for any $n$ and any $n$ dimensional (real or complex) Banach space $E$, there is an isomorphism $u: l_{2}^{n} \rightarrow E$ such that

$$
d_{k}(u) \leq b(\alpha)\left(\frac{n}{k}\right)^{\alpha} \text { and } c_{k}\left(u^{-1}\right) \leq b(\alpha)\left(\frac{n}{k}\right)^{\alpha}
$$

for $1 \leq k \leq n$, where $b(\alpha) \leq b_{0}\left(\alpha-\frac{1}{2}\right)^{-\frac{1}{2}}$ and $b_{0}>0$ is an absolute constant. 
Furthermore, for $0<\delta \leq 1$ and a non-increasing sequence of positive numbers $\left(s_{k}\right)_{k \in \mathbb{N}}$ we use the estimate

$$
\left(\prod_{k=1}^{n} s_{k}\right)^{\frac{1}{n}} \leq\left(\prod_{k=1}^{\left[\frac{n}{1+\delta}\right]} s_{[\delta k]+k-1}\right)^{\frac{1}{\left.\frac{n}{1+\delta}\right]}}, n \in \mathbb{N} .
$$

Moreover, the proof uses ideas presented in CHe91 and DJ93. If $\lambda_{n}(T)=0$, then there is nothing to prove. So we assume $\lambda_{n}(T) \neq 0$. By [K86], P87, or CS90] we can find an $n$-dimensional Banach space $X_{n}$ of $X$ invariant under $T$ such that the restriction $T_{n}$ of $T$ to $X_{n}$ has precisely $\lambda_{1}(T), \ldots, \lambda_{n}(T)$ as its eigenvalues. By Pisier we get for $\frac{1}{2}<\alpha \leq 1$ an isomorphism $u: l_{2}^{n} \rightarrow X_{n}$ such that

$$
d_{k}(u) \leq b(\alpha)\left(\frac{n}{k}\right)^{\alpha} \text { and } c_{k}\left(u^{-1}\right) \leq b(\alpha)\left(\frac{n}{k}\right)^{\alpha}
$$

for $1 \leq k \leq n$. Applying Weyl's inequality to the Hilbert space operator $u^{-1} T_{n} u$ and inserting the principle of related operators [P87] we arrive at

$$
\left(\prod_{k=1}^{n}\left|\lambda_{k}(T)\right|\right)^{\frac{1}{n}} \leq\left(\prod_{k=1}^{n} s_{k}\left(u^{-1} T_{n} u\right)\right)^{\frac{1}{n}}
$$

For estimating the right-hand side of the inequality we put $m:=\left[\frac{n}{1+\delta}\right]$ for $0<\delta \leq$ 1. Then we have

$$
\left(\prod_{k=1}^{n} s_{k}\left(u^{-1} T_{n} u\right)\right)^{\frac{1}{n}} \leq\left(\prod_{k=1}^{m} s_{[\delta k]+k-1}\left(u^{-1} T_{n} u\right)\right)^{\frac{1}{m}}
$$

The mixing multiplicativity (MI) (ii) and (iii) of an injective and surjective $s$ number sequence yields for the single $s$-numbers the estimate

$$
\begin{aligned}
s_{[\delta k]+k-1}\left(u^{-1} T_{n} u\right) & \leq c_{\left[\frac{\delta k}{2}\right]}\left(u^{-1}\right) s_{k}\left(T_{n}\right) d_{\left[\frac{\delta k}{2}\right]}(u) \\
& \leq b^{2}(\alpha)\left(\frac{n}{\left[\frac{\delta k}{2}\right]}\right)^{2 \alpha} s_{k}\left(T_{n}\right) \\
& \leq b^{2}(\alpha)\left(\frac{n}{\left[\frac{\delta k}{2}\right]}\right)^{2 \alpha} s_{k}(T) \text { for } 1 \leq k \leq m .
\end{aligned}
$$

Hence,

$$
\left(\prod_{k=1}^{m} s_{[\delta k]+k-1}\left(u^{-1} T_{n} u\right)\right)^{\frac{1}{m}} \leq b^{2}(\alpha)\left(\prod_{k=1}^{m} \frac{n}{\left[\frac{\delta k}{2}\right]}\right)^{\frac{2 \alpha}{m}}\left(\prod_{k=1}^{m} s_{k}(T)\right)^{\frac{1}{m}}
$$

From $\left[\frac{\delta k}{2}\right] \geq \frac{\delta k}{4}, m=\left[\frac{n}{1+\delta}\right] \geq \frac{n}{2(1+\delta)}$ and $e^{m} \geq \frac{m^{m}}{m !}$ we obtain for the first term on the right-hand side of the inequality the estimate

$$
\begin{aligned}
\left(\prod_{k=1}^{m} \frac{n}{\left[\frac{\delta k}{2}\right]}\right)^{\frac{2 \alpha}{m}} & \leq\left(\frac{4}{\delta}\right)^{2 \alpha} e^{2 \alpha}\left(\frac{n}{m}\right)^{2 \alpha} \\
& \leq\left(\frac{4}{\delta}\right)^{2 \alpha} e^{2 \alpha}(2(1+\delta))^{2 \alpha} \leq 16^{2} e^{2}\left(\frac{1}{\delta}\right)^{2 \alpha} .
\end{aligned}
$$


Combining the previous inequalities we arrive at

$$
\left(\prod_{k=1}^{n}\left|\lambda_{k}(T)\right|\right)^{\frac{1}{n}} \leq 16^{2} e^{2} b^{2}(\alpha)\left(\frac{1}{\delta}\right)^{2 \alpha}\left(\prod_{k=1}^{m} s_{k}(T)\right)^{\frac{1}{m}} .
$$

Finally, it remains to estimate the constant appearing on the right-hand side of the inequality by choosing an appropriate $\alpha, \frac{1}{2}<\alpha \leq 1$. To this end, first let $\frac{1}{e} \leq \delta \leq 1$. If we choose $\alpha=1$, then for the constant $c(\delta)$ we obtain $c(\delta) \leq 2 \cdot 16^{2} e^{4} b_{0}^{2}$. If $0<\delta \leq \frac{1}{e}$, then we choose $\alpha=\frac{1}{2}+\frac{1}{2 \ln \left(\frac{1}{\delta}\right)}$, which guarantees $\frac{1}{2}<\alpha \leq 1$ and for $c(\delta)$ we check that

$$
c(\delta) \leq 2 \cdot 16^{2} e^{3} b_{0}^{2} \frac{1}{\delta} \ln \left(\frac{1}{\delta}\right) .
$$

Summarizing the previous estimates we obtain

$c(\delta) \leq 2 \cdot 16^{2} e^{3} b_{0}^{2} \max \left\{e, \frac{1}{\delta} \ln \left(\frac{1}{\delta}\right)\right\} \leq 2 \cdot 16^{2} e^{4} b_{0}^{2}\left(1+\frac{1}{\delta} \ln \left(\frac{1}{\delta}\right)\right)$ for $0<\delta \leq 1$.

\section{REFERENCES}

[CHe91] B. Carl, A. Hess, Estimates of covering numbers. J. Approx. Theory 65 (1991), 121-139. MR.1104155 (93d:47042)

[CHi07] B. Carl, A. Hinrichs, Optimal Weyl type inequalities for operators in Banach spaces. Positivity 11 (2007), 41-55. MR2297321 (2007m:47036)

[CS90] B. Carl, J. Stephani, Entropy, Compactness and the Approximation of Operators. Cambridge University Press, 1990. MR.1098497 (92e:47002)

[DJ93] M. Defant, M. Junge, Some estimates on entropy numbers. Israel J. Math. 84 (1993) 417 - 433. MR1244678 (94j:47034)

[H05] A. Hinrichs, Optimal Weyl inequalities in Banach spaces. Proc Amer. Math. Soc. 134 (2005), 731 - 735. MR2180891 (2006f:47021)

[K86] H. König, Eigenvalue distribution of compact operators. Operator Theory: Advances and Applications 16, Birkhäuser, Basel, 1986. MR889455 (88j:47021)

[K01] H. König, Eigenvalues of operators and applications. Handbook of the Geometry of Banach Spaces, Vol. I, North-Holland, Amsterdam, 2001, 941 - 974. MR 1863710 (2003f:47035)

[KRT80] H. König, J. R. Retherford, N. Tomczak-Jaegermann, On the eigenvalues of $(p, 2)$ summing operators and constants associated to normed spaces. J. Funct. Anal. 37 (1980), 88 - 126. MR576647 (81m:47033)

[P80a] A. Pietsch, Weyl numbers and eigenvalues of operators in Banach spaces. Math. Ann. 247 (1980), 149 - 168. MR.568205(82i:47073a)

[P80b] A. Pietsch, Operator Ideals. North-Holland, Amsterdam, New York, Oxford, 1980. MR582655 (81j:47001)

[P87] A. Pietsch, Eigenvalues and $s$-Numbers. Cambridge Studies in Advanced Mathematics 13, Cambridge University Press, 1987. MR890520 (88j:47022b)

[Pi89a] G. Pisier, A new approach to several results of V. Milman. J. Reine Angew. Math. 393 (1989), 115 - 131. MR.972362 (90a:46038)

[Pi89b] G. Pisier, Volume Inequalities in the Geometry of Banach Spaces. Cambridge Univ. Press, 1989. MR1036275 (91d:52005)

Mathematisches Institut, FSU Jena, Ernst-Abbe-Platz 1-3, D-07743 Jena, Germany

E-mail address: carl@minet.uni-jena.de 